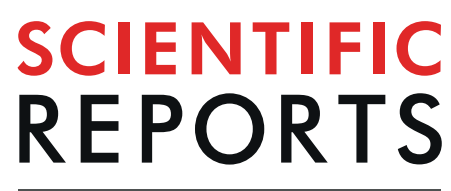

natureresearch

\title{
Regulation of histamine and diamine oxidase in patients undergoing orthotopic liver transplantation
}

Judith Schiefer ${ }^{1}$, Joanna Baron-Stefaniak (10 ${ }^{1}$, Thomas Boehm ${ }^{2}$, Patricia Wadowski ${ }^{2}$, Gabriela Berlakovich ${ }^{3}$, Lorenz Kuessel ${ }^{4}{ }^{4}$, Jakob Mühlbacher ${ }^{3}$, Petra Jilma-Stohlawetz ${ }^{5}$, Michael Schwameis ${ }^{6}$, Bernd Jilma ${ }^{2 *}$ \& Peter Faybik ${ }^{1}$

Increased concentrations of the vasodilator histamine have been observed in patients undergoing abdominal surgery. The role of histamine during orthotopic liver transplantation (OLT) has only been studied in animals. The aim of this study was to measure plasma concentrations of histamine and its degrading enzyme diamine oxidase (DAO) in patients undergoing orthotopic liver transplantation, and assess whether histamine or DAO correlate with intraoperative noradrenaline requirements. Histamine and DAO concentrations were measured in 22 adults undergoing liver transplantation and 22 healthy adults. Furthermore, norepinephrine requirements during liver transplantation were recorded. Baseline concentrations of histamine and DAO were greater in patients, who underwent liver transplantation, than in healthy individuals (Histamine: $6.4 \mathrm{nM}$, IOR[2.9-11.7] versus 4.3 $\mathrm{nM}$, IOR[3.77.1], $\mathrm{p}=0.029$; DAO: $2.0 \mathrm{ng} / \mathrm{mL}$, IOR[1.5-4.1] versus $<0,5 \mathrm{ng} / \mathrm{mL}, \mathrm{IOR}[<0.5-1.1], \mathrm{p}<0.001$ ). During liver transplantation, histamine concentrations decreased to $1.8 \mathrm{nM}, 1 \mathrm{IR}[0.5-4.9]$ in the anhepatic phase ( $p<0.0001$ versus baseline), and to $1.5 \mathrm{nM}$, IQR [0.5-2.9] after reperfusion ( $p<0.0001$ versus baseline). In contrast, DAO concentrations increased to $35.5 \mathrm{ng} / \mathrm{ml}, 1 \mathrm{IQR}[20-50]$ in the anhepatic phase $(p=0.001$ versus baseline) and to $39.5 \mathrm{ng} / \mathrm{ml}$, IQR [23-64] after reperfusion ( $p=0.001$ versus baseline), correlating inversely with histamine. Norepinephrine requirements during human liver transplantation correlated significantly with DAO concentrations in the anhepatic phase $(r=0.58, p=0.011)$ and after reperfusion $(r=0.56 ; p=0.022)$. In patients undergoing orthotopic liver transplantation, histamine concentrations decrease whereas DAO concentrations increase manifold. Diamine oxidase correlates with intraoperative norepinephrine requirements in patients undergoing OLT.

Orthotopic liver transplantation (OLT) is the only curative procedure for end-stage liver disease (ESLD). Patients with ESLD usually present with a systemic hyperdynamic cardiovascular regulation, demonstrating an increased cardiac output, reduced systemic vascular resistance and impaired vascular responsiveness to stress. During OLT, patients are exposed to further major hemodynamic alterations primarily due to temporary caval clamping, blood loss and ischemia/reperfusion (I/R) injury. Temporary clamping of the inferior vena cava decreases cardiac output by approximately $50 \%$ due to a reduction in venous return to the heart ${ }^{1,2}$, while blood loss leads to hypovolemia. I/R injury, mainly characterized by oxidative damage and a severe inflammatory response, triggers the secretion of vasoactive mediators into the recipients' systemic circulation ${ }^{3}$. Thus, OLT is frequently associated with hemodynamic instability of the recipient presenting clinically with hypotension, increased vasopressor support and cardiac arrhythmias.

${ }^{1}$ Department of Anesthesia, General Intensive Care and Pain Management, Medical University of Vienna, Waehringer Guertel 18-20, 1090, Vienna, Austria. 'Department of Clinical Pharmacology, Medical University of Vienna, Waehringer Guertel 18-20, 1090, Vienna, Austria. ${ }^{3}$ Department of Surgery, Division of Transplantation, Medical University of Vienna, Waehringer Guertel 18-20, 1090, Vienna, Austria. ${ }^{2}$ Department of Obstetrics and Gynecology, Medical University of Vienna, Waehringer Guertel 18-20, 1090, Vienna, Austria. ${ }^{5}$ Department of Laboratory Medicine, Division of Medical and Chemical Laboratory Diagnostics, Medical University of Vienna, Waehringer Guertel 18-20, 1090, Vienna, Austria. ${ }^{6}$ Department of Emergency Medicine, Medical University of Vienna, Waehringer Guertel 1820, 1090, Vienna, Austria. *email: bernd.jilma@meduniwien.ac.at 
Histamine is stored primarily in basophils and mast cells, and its release is triggered by various antigens, cytokines and drugs, but also by hypoxia and $\mathrm{I} / \mathrm{R}^{4}$. When released, histamine exerts pleiotropic physiological and pathophysiological regulatory functions, mainly inducing systemic vasodilation and increasing capillary permeability $^{3}$. Experimental studies have demonstrated that histamine concentrations increase in pigs undergoing OLT ${ }^{5}$. In clinical studies, increased plasma histamine concentrations have been observed in patients with cirrhosis ${ }^{6}$ and in patients undergoing abdominal surgeries ${ }^{7}$. However, no human studies have assessed histamine concentrations in patients undergoing OLT.

Balance between release and clearance of histamine is of utmost importance for hemodynamic stability in humans. In order to prevent or minimize an overshooting systemic vasoactive response to histamine, the body is equipped with various defense mechanisms to tightly control the plasma histamine concentrations ${ }^{6}$. Excessive enteral and likely renal re-absorption of histamine are prevented by the constitutive expression of the histamine metabolizing enzyme diamine oxidase $(\mathrm{DAO})^{8}$. Furthermore, the liver is a major organ responsible for clearing excessive histamine from the circulation ${ }^{9}$.

During OLT patients are exposed to an anhepatic period. We hypothesized that in patients undergoing OLT the anhepatic phase induces a dysbalance between release and clearance of histamine, contributing to systemic hypotension during OLT. Thus, the aim of this study was to measure concentrations of histamine and its degrading enzyme DAO in patients undergoing OLT, and to correlate histamine and DAO concentrations with the intra-operative noradrenaline requirements.

\section{Materials and Methods}

Study subjects. This single-center, observational study was performed in accordance with the ethical standards laid down in the Declaration of Helsinki. Institutional ethics committee (Medical University of Vienna) approval for the study was obtained. Written informed consent was obtained before enrollment into the study.

Twenty-two adult patients scheduled for OLT at the General Hospital of Vienna between August 2014 and August 2015 were enrolled in the study. Exclusion criteria were combined liver-kidney or liver-lung transplantation, and the need of intraoperative veno-venous bypass ${ }^{10}$. To allow direct comparison between patients with ESLD and healthy individuals, blood samples were additionally obtained form twenty-two healthy age-matched adult volunteers.

Anesthesia, surgery, and immunosuppression. Anesthesia and surgery were performed according to the local standards, which have already been previously described by our research group ${ }^{10,11}$. The surgical technique was performed pursuant to the local standard technique with cross clamping of the inferior caval vein. All grafts were $\mathrm{ABO}$ compatible and matched for the graft-to-recipient-weight ratio. Immunosuppression was initiated already intraoperatively, before graft reperfusion, and continued according to the local standardized protocol for immunosuppression in OLT.

Data and sample collection. Demographic data, primary disease leading to ESLD, the model of end-stage liver disease (MELD) score, surgical reports, the anesthetic report, as well as relevant parameters from the postoperative course on the intensive care unit, including the use of blood and coagulation products were collected for each patient. Diagnosis of clinically significant portal hypertension was established in patients suffering from ESLD fulfilling one or more of the following: hepatic vein pressure gradient $>10 \mathrm{mmHg}$, gastroenteral varices, liver stiffness measurement or imaging showing collateral circulation ${ }^{12,13}$.

Results from routinely daily measured laboratory parameters were extracted from the patient data management software (PDMS, Phillips, USA). The maximal norepinephrine infusion rate and the total amount of infused norepinephrine during OLT were recorded. In patients scheduled for OLT, blood samples were drawn from an arterial line, which is placed standardly during OLT. Arterial blood samples were collected at the following pre-defined time points (TP): at baseline (BL) prior to surgery, during OLT in the anhepatic phase immediately prior to reperfusion (TP1) and 10 minutes after graft - reperfusion (TP2). Control blood samples were obtained in the morning from the radial artery of fasting adult volunteers. Immediately after collection, plasma samples were centrifuged at $2600 \mathrm{~g}$ for 20 minutes and stored at $-80^{\circ} \mathrm{C}$ until analysis.

Sample analysis. Histamine concentrations in plasma were measured using a commercially available enzyme-linked immunosorbent assay (ELISA) (Immunotech, Beckmann Coulter Company, Brea, CA) on a standard curve ranging from 1-90 $\mathrm{nM}$.

Diamine oxidase concentrations were measured using a custom-assembled ELISA as described recently ${ }^{14}$ using recombinant human diamine oxidase as a standard ${ }^{15}$. Briefly, a purified monoclonal antibody raised against human DAO isolated from Caco- 2 cell supernatant was coated at $5 \mu \mathrm{g} / \mathrm{ml}$ onto white high protein binding microtiter plates (Greiner bio-one 655074) in $50 \mathrm{mM}$ carbonate-bicarbonate coating buffer $\mathrm{pH} 9.6$ overnight at $4{ }^{\circ} \mathrm{C}$. EDTA plasma was diluted 1 to 5 with LowCross-Buffer (CANDOR bioscience 1000500; Germany). Bound human DAO was detected with an anti-DAO polyclonal rabbit IgG serum fraction diluted 1 to 1000 . The bound rabbit antibodies were detected with a 1 to 32000 dilution of donkey anti-rabbit IgG HRP-labelled antibodies (Sigma SAB3700928; Austria). Antibodies were incubated for 50 minutes at room temperature. Bound HRP molecules were quantified with the SuperSignal ${ }^{\mathrm{TM}}$ ELISA Pico Chemiluminescent Substrate (Thermo Scientific 37070; Vienna) using a standard chemiluminescent reader (Victor ${ }^{\mathrm{TM}} 1420$ Microtiter Plate Reader (Perkin Elmer). The limit of blank (LOB) and detection (LOD) are 0.27 and $0.48 \mathrm{ng} / \mathrm{ml}$ respectively using 42 standard curve determinations. The estimate limit of quantification (eLOQ) is $0.70 \mathrm{ng} / \mathrm{ml}$. A value of $0.48 \mathrm{ng} / \mathrm{mL}$ was assigned for values below the detection limit for conservative statistical comparisons.

Mast cell degranulation triggered by anaphylactic reactions is a frequent cause for histamine release into patient's systemic circulation ${ }^{16}$, but may also be associated with DAO release ${ }^{17}$. To exclude possible intraoperative 


\begin{tabular}{|l|l|}
\hline Age (years) & $57(29-72)$ \\
\hline Sex (female/male), $\mathrm{n}$ & $7 / 15$ \\
\hline MELD score & $17(9-40)$ \\
\hline Child Pugh Score, $\mathrm{n}(\mathrm{A} / \mathrm{B} / \mathrm{C})$ & $(1 / 8 / 13)$ \\
\hline Etiology of liver disease & $\mathbf{N}(\%)$ \\
\hline Alcohol & $7(31 \%)$ \\
\hline Hepatocellular carcinoma & $3(13 \%)$ \\
\hline Alcohol and hepatocellular carcinoma & $1(5 \%)$ \\
\hline Alcohol and viral & $1(5 \%)$ \\
\hline Viral and hepatocellular carcinoma & $2(9 \%)$ \\
\hline Cholestatic liver disease & $2(9 \%)$ \\
\hline Cryptogenic cirrhosis & $2(9 \%)$ \\
\hline Autoimmune cirrhosis & $2(9 \%)$ \\
\hline Acute Liver Failure & $1(5 \%)$ \\
\hline Re-OLT & $1(5 \%)$ \\
\hline Comorbidities & $\mathbf{N}(\%)$ \\
\hline Diabetes mellitus & $7(32 \%)$ \\
\hline Cardiovascular & $8(36 \%)$ \\
\hline Chronic kidney disease & $3(14 \%)$ \\
\hline
\end{tabular}

Table 1. Demographic data and indications of patients undergoing liver transplantation. Categorical variables are described by absolute and relative frequencies or median and IQR, if applicable. Abbreviations: IQR, interquartile range; MELD, model for end-stage liver disease.

mast cell degranulation as a potential cause for histamine release in our patients, total tryptase concentrations were measured in plasma of patients undergoing OLT (ImmunoCAP Tryptase ELISA Kit, Thermoscientific, Waltham, MA).

Diamine oxidase release is triggered by heparin ${ }^{18}$. To determine whether endogenous hepariniziation could explain a possible DAO release, we measured aPTT and anti-FXa activities in a subgroup of patients, and performed a thrombelastometric analysis calculating the difference between measurements with and without heparinase (INTEM - HEPTEM).

Statistical analysis. Statistical analyses were performed using Prism 6.0 (GraphPad Software, La Jolla, CA) and Statistica, (Stat Soft Inc., Tulsa, OK). Results are depicted as median with interquartile ranges (IQR, [25-75\%], unless indicated otherwise. A Kolmogorov-Smirnov test was used to verify normal distribution. A Friedman analysis of variance (ANOVA), followed by post-hoc Wilcoxon tests, and U-tests were applied for comparison between groups. Correlations were calculated by the Spearman ranks correlation analysis. Receiver operating characteristic (ROC) curve was used to assess the diagnostic value of plasma histamine and DAO concentrations to discriminate the need for higher dosage of vasopressor support with norepinephrine $(\geq 0.3 \mathrm{mcg} / \mathrm{kg} /$ min) during the anhepatic phase and reperfusion period. Statistical significance was set at $p<0.05$.

Sample size calculation. Previous studies assessed plasma histamine concentrations in healthy volunteers ${ }^{19}$, and compared histamine concentrations between cirrhotic patients and healthy individuals ${ }^{20}$. Based on these data we estimated an intra- and inter-subject coefficient of variation of plasma histamine concentrations of approximately $60 \%$ and $90 \%$, respectively. We calculated that 19 subjects in each group would be sufficient to allow detection of a 2 -fold greater histamine concentration in patients undergoing OLT with $80 \%$ power and an alpha error of $<5 \%$. We felt that this effect size is clinically justified because plasma histamine concentrations greater than 2-fold the upper normal limit of $11 \mathrm{nM}$ might be hemodynamically relevant as demonstrated by a $30 \%$ increase in the heart rate in healthy volunteers ${ }^{3}$.

Ethics declaration. Approval was obtained from the local ethics committee of the Medical University of Vienna. Patients informed consent was obtained before enrollment in the study.

\section{Results}

Demographic data. Twenty-one patients (mean age $56 \pm 12$ years) with ESLD undergoing OLT were enrolled in the study. In addition, one patient with acute liver failure was enrolled. Furthermore, we included 22 healthy age-matched volunteers. Table 1 shows the demographic data and etiology leading to OLT. Table 2 depicts baseline and postoperative laboratory parameters after OLT. Table 3 shows the intraoperative substitution of blood products and crystalloids.

Perioperative plasma histamine concentrations. Median histamine concentrations at BL in all patients scheduled for OLT were significant greater than those in healthy control individuals (6.4 nM, IQR [2.9-11.7] vs. $4.3 \mathrm{nM}, \mathrm{IQR}$ [3.7-7.1]; $\mathrm{p}=0.029)$. In patients undergoing OLT, plasma histamine concentrations decreased to $1.8 \mathrm{nM}$, IQR [0.5-4.9] at TP1 in the anhepatic phase ( $\mathrm{p}<0.001$ versus $\mathrm{BL}$ ) and to $1.5 \mathrm{nM}, \mathrm{IQR}$ [0.5-2.9] after reperfusion ( $\mathrm{p}<0.001$ versus BL; Fig. 1). In 21 patients with ESLD undergoing OLT median plasma histamine 


\begin{tabular}{|l|l|l|l|}
\hline Parameter & Pre-OLT & Post-OLT & Day 1 post OLT \\
\hline Hemoglobin, $(\mathrm{g} / \mathrm{dL})$ & $10.8(7.4-15.3)$ & $9.0(7.5-11.3)$ & $8.8(5.7-12)$ \\
\hline White blood cells, $\left(10^{9} / \mathrm{L}\right)$ & $3.7(1.3-7.3)$ & $7.9(0.7-17.2)$ & $8.2(1.1-34.2)$ \\
\hline Platelets, $\left(10^{9} / \mathrm{L}\right)$ & $63(16-239)$ & $47(21-183)$ & $52(18-185)$ \\
\hline AST, $(\mathrm{U} / \mathrm{L})$ & $46(21-336)$ & $1085(398-10430)$ & $557(91-16620)$ \\
\hline ALT, $(\mathrm{U} / \mathrm{L})$ & $31(16-229)$ & $939(384-3961)$ & $784(266-8100)$ \\
\hline Bilirubin, $(\mathrm{mg} / \mathrm{dL})$ & $2.9(0.5-41.7)$ & $4.5(1-19.3)$ & $3.6(0.8-20.1)$ \\
\hline Creatinine, $(\mathrm{mg} / \mathrm{dL})$ & $0.8(0.4-4.4)$ & $1.4(0.6-5)$ & $1.7(0.7-5.8)$ \\
\hline Prothrombin time, $(\%)$ & $41(5-89)$ & $43(24-129)$ & $47(14-88)$ \\
\hline aPTT, $(\mathrm{s})$ & $42(35-97)$ & $91(44-173)$ & $46(36-68)$ \\
\hline
\end{tabular}

Table 2. Perioperative laboratory parameters. Data are given as median and IQR. Abbreviations: ALT, alanine aminotransferase; aPTT, activated partial thromboplastin time; AST, aspartate aminotransferase; IQR, interquartile range; OLT, orthotopic liver transplantation.

\begin{tabular}{|l|c|}
\hline \multicolumn{2}{|l|}{ Blood products given } \\
\hline Packed red blood cells, (units) & $3(0-16)$ \\
\hline Fresh frozen plasma, (units) & $6(0-20)$ \\
\hline Platelets, (units) & $1(0-4)$ \\
\hline Crystalloids, (ml) & $5625(2500-9000)$ \\
\hline Colloids, (ml) & $315(0-600)$ \\
\hline Prothrombin complex concentrates, (units) & $1500(0-6500)$ \\
\hline
\end{tabular}

Table 3. Intraoperative substitution of blood products and crystalloids. Data are given as median and IQR. Abbreviations: IQR, interquartile range.
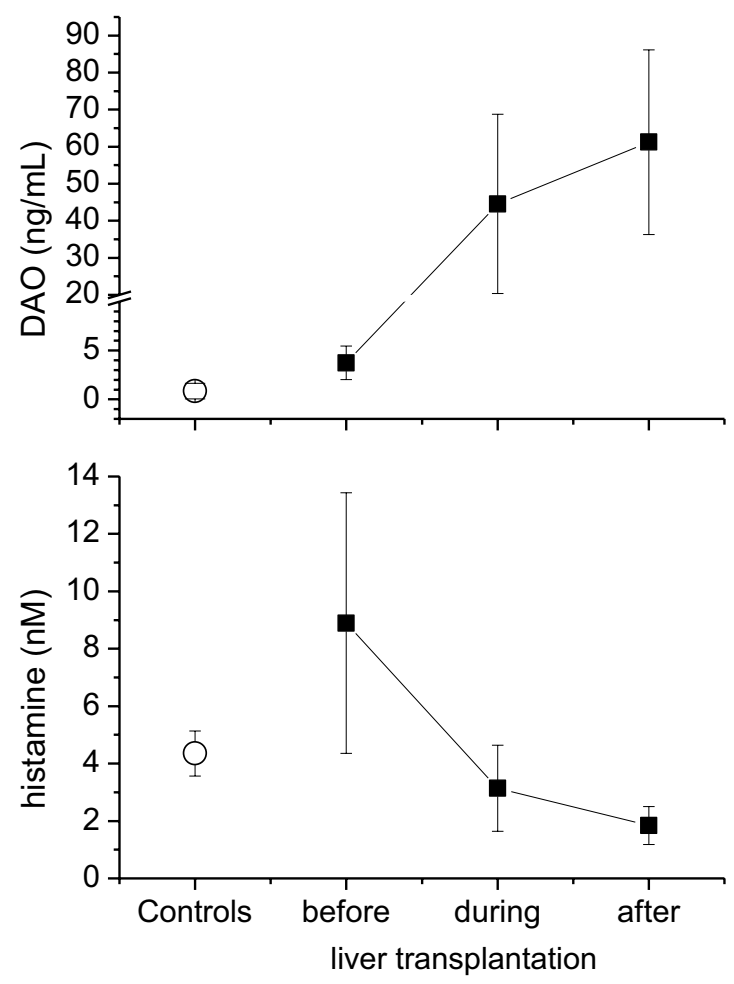

Figure 1. Median plasma concentrations of histamine and DAO. Concentrations of (A) plasma histamine and (B) plasma DAO in healthy controls, and patients undergoing OLT at 3 different time points: baseline (BL), in the anhepatic phase (TP1), and after reperfusion (TP2).

concentrations decreased from 6.6 nM (IQR 3.3-11.8) at BL to 1.88 nM (IQR 0.5-5.1) during anhepatic phase $(\mathrm{p}<0.001$ versus $\mathrm{BL})$ and further to $1.82 \mathrm{nM}$ (IQR $0.5-2.9)$ after reperfusion ( $\mathrm{p}<0.001$ versus $\mathrm{BL})$. In the only 1 patient with acute liver failure undergoing OLT, plasma histamine concentrations decreased from $2.1 \mathrm{nM}$ at BL to $0.6 \mathrm{nM}$ during the anhepatic phase and to $0.5 \mathrm{nM}$ after reperfusion. Interestingly, histamine concentrations 
measured after reperfusion were lower in patients undergoing OLT than histamine concentrations of healthy volunteers at BL. Neither the amount and type of fluids, nor the number of blood products administered during transplantation correlated with plasma histamine concentrations.

Perioperative plasma DAO concentrations. In patients scheduled for OLT, baseline plasma DAO concentrations were significantly greater $(2.0 \mathrm{ng} / \mathrm{mL}$, IQR $[1.5-4.1])$ than in age-matched controls $(<0.50 \mathrm{ng} / \mathrm{mL}$, IQR $[<0.5$ to 1.1$] ; \mathrm{p}<0.001)$. In patients undergoing OLT, DAO concentrations increased to $35.5 \mathrm{ng} / \mathrm{mL}, \mathrm{IQR}$ [20-50] in the anhepatic phase ( $\mathrm{p}=0.001$ versus baseline), and to $39.5 \mathrm{ng} / \mathrm{mL}$, IQR [23-64] in the reperfusion period ( $\mathrm{p}=0.001$ versus baseline) (Fig. 1). Of note, DAO concentrations correlated inversely with histamine concentrations in the anhepatic phase $(\mathrm{r}=-0,68 ; \mathrm{p}=0.001)$ and during reperfusion $(\mathrm{r}=-0.53, \mathrm{p}=0.02)$. In patients with ESLD undergoing OLT, median DAO concentrations increased from $2.1 \mathrm{ng} / \mathrm{mL}$ (IQR $1.5-4.0)$ at $\mathrm{BL}$ to $36.2 \mathrm{ng} / \mathrm{mL}$ (IQR 20.4-50.2) in the anhepatic phase ( $\mathrm{p}=0.001$ versus baseline), and further to $41.6 \mathrm{ng} /$ $\mathrm{mL}$ (IQR 24.6-64.3) in the reperfusion period ( $\mathrm{p}=0.001$ versus baseline). In one patient with acute liver failure undergoing OLT, DAO concentrations increased from $17.5 \mathrm{ng} / \mathrm{ml}$ at BL to $20.4 \mathrm{ng} / \mathrm{mL}$ in the anhepatic phase, and later decreased to $12.6 \mathrm{ng} / \mathrm{mL}$ in the reperfusion period. Neither the amount and type of fluids, nor the number of blood products administered during transplantation correlated with DAO concentrations.

Effect of liver function on plasma histamine and DAO concentrations. There was no statistically significant correlation between MELD score, prothrombin time, serum albumin or total bilirubin with plasma histamine or DAO concentrations at baseline. Furthermore, no significant correlation was found between the plasma histamine or DAO concentrations and AST as a marker of I/R injury at the end of OLT or on day 1 after transplantation.

Effect of clinical significant portal hypertension on plasma histamine and DAO concentrations. Seventeen patients (80\%) with ESLD included in our study fulfilled the criteria of clinically significant portal hypertension, $4(20 \%)$ patients did not. Plasma histamine $(\mathrm{p}=0.6)$ and DAO $(\mathrm{p}=0.33)$ concentration were not significantly different between patients with or without clinical significant portal hypertension at baseline before OLT. The median plasma histamine concentration during reperfusion was significantly higher in patients with clinical significant portal hypertension than in patients without $(2.4 \mathrm{nM}, \mathrm{IQR}$ [0.5-3.1] vs. $0.54 \mathrm{nM}$, IQR [0.5-0.5]; $\mathrm{p}=0.03)$. However, there were no statistically significant differences in plasma histamine or DAO concentrations at any other time-point between patients with or without signs of clinical significant portal hypertension during our study.

Norepinephrine requirements and concentrations of histamine and DAO during OLT. During OLT, the median norepinephrine infusion rate was $0.287 \mathrm{mcg} / \mathrm{kg} / \mathrm{min}, \mathrm{IQR}$ [0.1-1.0]. The median cumulative norepinephrine dose administered during OLT was $4.5 \mathrm{mg}$ [range 1.3-20.0]. There was no significant correlation between plasma histamine concentrations and norepinephrine requirements. Interestingly, the maximal intraoperative norepinephrine infusion rate correlated significantly with DAO concentrations in the anhepatic phase $(\mathrm{r}=0.58 ; \mathrm{p}=0.011)$ and after reperfusion $(\mathrm{r}=0.56 ; \mathrm{p}=0.022)$. The total intra-operative norepinephrine dose correlated significantly with DAO concentrations in the anhepatic phase $(r=0.46 ; p=0.049)$, but not in the reperfusion phase $(r=0.38 ; \mathrm{p}=0.11)$. In the ROC curve analysis, DAO during the anhepatic phase exhibited an AUC of 0.85 (95\% confidence interval, $0.62-0.97 ; \mathrm{p}=0.0001)$ for the norepinephrine requirement of at least $0.3 \mathrm{mcg} / \mathrm{kg} / \mathrm{min}$ or higher. The sensitivity and specifity for DAO in the anhepatic phase with an optimal cut-off value of greater than $33.4 \mathrm{ng} / \mathrm{mL}$ were $80 \%$ and $89 \%$, respectively. Results of ROC curve analysis of DAO in the reperfusion period and plasma histamine in the anhepatic phase and after reperfusion were of poor prognostic value to predict higher vasopressor support. In our patient population, the duration of surgery did not significantly correlate with cumulative norepinephrine use.

Tryptase release and endogenous heparinization. To assess whether changes in histamine concentrations were due to increased mast cell activity occurring during OLT, plasma-tryptase concentrations were quantified. In all except one patient, plasma-tryptase concentrations remained stable after induction of anesthesia $(4.3 \mu \mathrm{g} / \mathrm{l}, \mathrm{IQR}[2.6-6.8]$ vs. $4.1 \mu \mathrm{g} / \mathrm{l}$, IQR [2.6-6.3]. In one patient, plasma-tryptase concentrations increased by $82 \%$ from baseline, fulfilling the criteria for mast cell activation ${ }^{21}$. In this patient, we measured the highest plasma histamine concentration prior to skin incision ( $48 \mathrm{nM}$; i.e. $>3$ SDs above the median of OLT patients included into this study).

In 4 out of 5 patients undergoing OLT, the anti-FXa activity increased from $<0.1 \mathrm{U} / \mathrm{mL}$ at baseline to $0.1 \mathrm{U} /$ $\mathrm{mL}$ [0.1-0.75] in the anhepatic phase. In addition, in all patients aPTT increased significantly in comparison to baseline, and delayed clotting was observed in thromboelastometry without heparinase. Taken together, these results suggest endogenous heparinization in patients undergoing OLT.

\section{Discussion}

The present study examined the regulation of histamine and its degrading enzyme DAO in patients undergoing OLT, and assessed whether histamine or DAO correlate with norepinephrine requirements during OLT. Baseline histamine and DAO concentrations were significantly elevated in patients undergoing OLT. Plasma histamine concentrations markedly decreased during OLT, whereas DAO concentrations increased manifold, which correlated with the intraoperative norepinephrine requirements during OLT.

Previous studies have demonstrated that plasma histamine concentrations were greater in cirrhotic patients compared to healthy controls ${ }^{6,20}$. Our findings are in agreement and extend those findings to ESLD patients scheduled for OLT. Greater plasma histamine concentrations in patients with ESLD likely result from reduced 
hepatic clearance of histamine in advanced liver disease ${ }^{22}$. In the clinical setting of OLT an anhepatic period is required, where the hepatic clearance of histamine is completely abolished. Therefore, it would be expected that histamine plasma concentrations increase during OLT. Surprisingly, plasma histamine concentrations decrease during the anhepatic phase and after reperfusion in patients undergoing OLT when compared to histamine concentrations at baseline. This may partially be due to intravenous administration of $40 \mathrm{mg}$ dexamethasone before the anhepatic phase as part of immunosuppression protocol in our patients. In fact, there is no sufficient literature about effects of steroids on histamine and DAO levels in patients suffering from ESLD or undergoing OLT. Dunsky et al. described in their study that corticosteroids induce a prominent decrease in leucocyte histamine due to a depletion of basophils without a decrease in histamine content per basophil in atopic subjects ${ }^{23}$. Another study in newborn rat intestine suggested that DAO activity may be induced by hydrocortisone ${ }^{24}$. Elevated DAO levels observed in our patients, and consequently raised metabolism of histamine may have further decreased plasma histamine concentrations.

In contrast to our results, Lorenz et al. demonstrated that plasma histamine concentrations increase in pigs exposed to experimental liver transplantation ${ }^{25}$. However, in contrast to human OLT, no steroids have been administered at any time in this study. Another study showed that systemic histamine concentrations increase in patients undergoing major abdominal surgeries. The increase in histamine concentrations was explained by administration of anesthetic drugs, infusion of plasma and manipulation of the gut ${ }^{7}$. Although similar triggers for histamine release suggested by Lorenz et al. were also present during OLT in our patients, the systemic histamine concentration decreased. Some explanations exist for these contradicting results. Firstly, different anesthetics and muscle relaxants, which are not in routine clinical use anymore, were used by Lorenz et al. Histamine release by older anesthetics and muscle relaxants was a well-described phenomenon, and could be reduced by the development of newer anesthetics and muscle relaxants such as propofol, sevoflurane and rocuronium, which were used in our study. In addition, the porcine OLTs described by Lorenz et al. were performed on porto-jugular bypass ${ }^{25}$, which was not the case in our study. Furthermore, in the study of Lorenz et al., abdominal surgeries were performed without caval clamping ${ }^{7}$. Of note is, that physiological alterations largely differ between surgeries performed in bicaval clamping without bypass and those performed with bypass or without bicaval clamping. These varying physiological conditions might serve as an explanation for different histamine secretion patterns between studies.

This is the first systematic investigation demonstrating elevated concentrations of DAO in patients with liver disease. Of note, DAO is rapidly metabolized by rat liver ${ }^{26}$. Thus, the increased baseline concentration of DAO might be explained by a decreased clearance of DAO in patients with ESLD. The most striking finding of this study was the manifold increase in DAO concentrations during OLT comparable in magnitude to early pregnancy ${ }^{27}$, and the likely resultant inverse correlation between DAO and histamine. The increase in systemic concentration of DAO during OLT can explain the degradation of histamine. We have recently shown that plasma levels of DAO (29-52 ng/mL) comparable to those observed in the anhepatic phase can rapidly lower plasma histamine concentrations by $\sim 50-85 \%$ within 10 minutes $^{27}$. Thus, the increase in DAO is a mechanistic explanation for the observed decrease in histamine concentrations in patients undergoing OLT.

A decrease in the hepatic clearance of DAO during the anhepatic phase is conceivably a major contributor to the acute and massive rise in DAO. However, several other factors may increase the release of diamine-oxidase during OLT. Heparin is known to release DAO into the systemic circulation ${ }^{18,28,29}$, and it has been described that heparin metabolism is impaired in liver failure ${ }^{30}$. Our results suggest that endogenous heparinization is present in patients undergoing OLT. Thus, the observed increase in systemic DAO concentration during OLT might be explained by the absence of hepatic clearance of heparin during the anhepatic phase ${ }^{30-32}$. Moreover, prothrombin complex concentrates (PCC) contain variable concentrations of heparin ${ }^{33}$, and administration of PCC during OLT could increase systemic heparin concentrations. The elevated heparin concentrations after administration of PCC could trigger the secretion of DAO. However, we found substantial increases in DAO concentrations in patients not receiving PCCs, making an association between administration of PCC and elevated DAO concentrations unlikely. Another pathophysiological pathway contributing to the gradual increase of systemic DAO concentration during OLT might be the DAO release from the gut. Cross clamping of the caval vein leads to a reduction of the cardiac output and subsequently to an intestinal hypoperfusion with venous congestion, representing a period of temporary intestinal ischemia ${ }^{34}$. The following breakdown of the intestinal mucosal barrier is associated with a high DAO release from the intestinal mucosa into the circulation ${ }^{24,35}$. Taken together, the increased DAO concentrations observed in patients undergoing OLT might be a net effect of reduced hepatic DAO clearance, increased heparin induced DAO release and DAO release from the gut injured by transient hypoperfusion during OLT.

Histamine plasma concentrations as low as $11 \mathrm{nM}$ increase the heart rate by approximately $15 \%$ and widen the gap between systolic and diastolic pressure by $10 \%$ in healthy humans ${ }^{3}$. Experimental studies yield conflicting results when assessing the association between histamine and cardiovascular changes during liver transplantation. While Hansen et al. proposed that histamine does not contribute to arterial hypotension after revascularization during OLT in pigs ${ }^{5}$, Lorenz et al. demonstrated that administration of antihistaminergic drugs during porcine OLT diminished arterial hypotension in the revascularization phase ${ }^{25,36}$. In the current study, histamine concentrations did not correlate with the norepinephrine requirements in patients undergoing OLT. Uniformly low levels of histamine due to degradation by DAO may have contributed to that finding. In contrast, we found that plasma DAO concentrations correlate with the norepinephrine requirements in the anhepatic phase and after revascularization during OLT. Norepinephrine is used to counteract hypotension which can result in or reflect intestinal hypoxemia, which may itself trigger DAO release. For example, hemorrhagic shock increased DAO levels 6-fold in rabbits ${ }^{27}$, and an hour of intestinal ischemia increased DAO concentrations 3.6-fold in rats ${ }^{24}$. Such an increase in DAO is conceivably accentuated in the presence of decreased clearance of DAO during the 
anhepatic phase. Interestingly, DAO levels also rise 100 -fold in anaphylactic shock in mastocytosis patients ${ }^{17}$, so that increased DAO release levels may partly reflect intestinal ischemia.

The current study has some limitations; the sample size was relatively small and therefore does not allow meaningful subgroup analysis according to the underlying etiology. Detailed correlation analysis between measured histamine and DAO concentrations and hemodynamic variables such as heart rate, mean arterial pressure, systemic vascular resistance or cardiac output could not be determined. Thus, we were unable to assess a contribution of histamine or DAO to compromised hemodynamics in patients undergoing OLT. Furthermore, the concentrations of histamine, DAO and tryptase during OLT could be underestimated due dilution by intraoperative crystalloid and colloid administration. Finally, this study did not directly quantify hepatic histamine clearance, since we have only measured histamine concentrations in the systemic circulation and not in the portal vein or the hepatic artery. Despite these limitations, this is the first study assessing concentrations of histamine and DAO in patients undergoing OLT. Our findings demonstrating an inverse correlation between histamine and DAO and the association between DAO and norepinephrine requirements in patients undergoing OLT are novel, and underline that the balance between histamine and DAO is of utmost importance for hemodynamic stability. Further studies assessing the significance of DAO in the clinical and experimental setting of OLT and other major surgeries should be promoted.

\section{Conclusion}

The results of this study show that patients with ESLD undergoing OLT have elevated baseline plasma concentrations of histamine and its degrading enzyme DAO. During OLT, plasma histamine concentrations markedly decreased, whereas DAO concentrations increased manifold. The increase in diamine oxidase is associated with hemodynamic compromise as measured by norepinephrine requirements in patients undergoing OLT.

\section{Data availability}

The datasets generated during and/or analyzed during the current study are available from the corresponding author on reasonable request.

Received: 24 September 2019; Accepted: 6 January 2020;

Published online: 21 January 2020

\section{References}

1. Zadrobilek, E., Mauritz, W., Feil, W., Wenzl, E. \& Sporn, P. Hemodynamic and metabolic changes during orthotopic liver transplantation. Anaesthesist 37(11), 672-9, http://www.ncbi.nlm.nih.gov/pubmed/3063132, Hamodynamische und metabolische Anderungen wahrend orthotoper Lebertransplantation (Nov 1988).

2. Estrin, J. A. et al. Hemodynamic changes on clamping and unclamping of major vessels during liver transplantation. Transplant Proc 21(3), 3500-5, http://www.ncbi.nlm.nih.gov/pubmed/2662498 (Jun 1989).

3. Kaliner, M., Sigler, R., Summers, R. \& Shelhamer, J. H. Effects of infused histamine: analysis of the effects of H-1 and H-2 histamine receptor antagonists on cardiovascular and pulmonary responses. J Allergy Clin Immunol 68(5), 365-71, http://www.ncbi.nlm.nih. gov/pubmed/7299000 (Nov 1981).

4. Kusche, J. et al. Intestinal diamine oxidase and histamine release in rabbit mesenteric ischemia. Gastroenterology 80 (5 pt 1), 980-7, http://www.ncbi.nlm.nih.gov/pubmed/6162712 (May 1981).

5. Hansen, C. P., Man, W. K., Kirkegaard, P., Jensen, S. L. \& Boesby, S. Changes in plasma histamine during orthotopic liver transplantation in the pig. Agents Actions 23(3-4), 348-50, http://www.ncbi.nlm.nih.gov/pubmed/3293368 (Apr 1988).

6. Stopik, D., Hampel, K. E. \& Beger, H. G. Plasma histamine concentration and hemodynamic changes in liver cirrhosis (author's transl). Leber Magen Darm 8(2), 91-3, http://www.ncbi.nlm.nih.gov/pubmed/634019, Plasmahistamingehalt und veranderte Hamodynamik bei Leberzirrhosen (Apr 1978).

7. Lorenz, W. et al.. Elevated plasma histamine concentrations in surgery: causes and clinical significance. Klin Wochenschr 52(9), 419-25, https://www.ncbi.nlm.nih.gov/pubmed/4137645 (May 1, 1974).

8. Beall, G. N. \& Vanarsdel, P. P. Jr. Histamine metabolism in human disease. J Clin Invest 39, 676-83, https://doi.org/10.1172/ JCI104083 (Apr 1960).

9. Mitchell, R. G., Butt, H. R. \& Code, C. F. Histamine metabolism in diseases of the liver. J Clin Invest 33(9), 1199-1203, https://doi. org/10.1172/JCI102993 (Sep 1954).

10. J. Baron-Stefaniak, et al. Comparison of macrophage migration inhibitory factor and neutrophil gelatinase-associated lipocalin-2 to predict acute kidney injury after liver transplantation: An observational pilot study. PLoS One 12(8), e0183162, https://doi. org/10.1371/journal.pone.0183162 (2017).

11. Schiefer, J. et al. Alterations of Endothelial Glycocalyx During Orthotopic Liver Transplantation in Patients With End-Stage Liver Disease. Transplantation 99(10), 2118-23, https://doi.org/10.1097/TP.0000000000000680 (Oct 2015).

12. de Franchis, R. \& Baveno, V. I. F. Expanding consensus in portal hypertension: Report of the Baveno VI Consensus Workshop: Stratifying risk and individualizing care for portal hypertension. J Hepatol 63(3), 743-52, https://doi.org/10.1016/j.jhep.2015.05.022 (Sep 2015).

13. Reiberger, T. et al. Austrian consensus guidelines on the management and treatment of portal hypertension (Billroth III). Wien Klin Wochenschr 129(Suppl 3), 135-158, https://doi.org/10.1007/s00508-017-1262-3 (Nov 2017).

14. Boehm, T. et al. Quantification of human diamine oxidase. Clin Biochem 50(7-8), 444-451, https://doi.org/10.1016/j. clinbiochem.2016.12.011 (May 2017).

15. Gludovacz, E. et al. Characterization of recombinant human diamine oxidase (rhDAO) produced in Chinese Hamster Ovary $(\mathrm{CHO})$ cells. J Biotechnol 227, 120-130, https://doi.org/10.1016/j.jbiotec.2016.04.002 (Jun 10 2016).

16. Vitte, J. Human mast cell tryptase in biology and medicine. Mol Immunol 63(1), 18-24, https://doi.org/10.1016/j. molimm.2014.04.001 (Jan 2015).

17. Boehm, T. et al. Massive release of the histamine-degrading enzyme diamine oxidase during severe anaphylaxis in mastocytosis patients. Allergy 74(3), 583-593, https://doi.org/10.1111/all.13663 (Mar 2019).

18. D’Agostino, L., et al. Plasma diamine oxidase (DAO) and heparin. Dig Dis Sci 29(11), 1070-1, http://www.ncbi.nlm.nih.gov/ pubmed/6435979 (Nov 1984).

19. Schwameis, M. et al. D-dimer and histamine in early stage bacteremia: A prospective controlled cohort study. Eur J Intern Med 26(10), 782-6, https://doi.org/10.1016/j.ejim.2015.10.024 (Dec 2015).

20. Stopik, D., Beger, H. G. \& Hampel, K. E. Studies on the pre- and posthepatic plasma histamine concentrations and their possible pathophysiological effects in liver cirrhosis (author's transl). Klin Wochenschr 56(5), 241-6, http://www.ncbi.nlm.nih.gov/ 
pubmed/633778, Untersuchungen uber die pra- und posthepatischen Plasmahistaminkonzentrationen und ihre moglichen pathophysiologischen Auswirkungen bei Lebercirrhose (Mar 1 1978).

21. Valent, P. et al. Definitions, criteria and global classification of mast cell disorders with special reference to mast cell activation syndromes: a consensus proposal. Int Arch Allergy Immunol 157(3), 215-25, https://doi.org/10.1159/000328760 (2012).

22. Kobayashi, Y. \& Schayer, R. W. In vitro demonstration of a new histamine-metabolizing enzyme. Arch Biochem Biophys 63(2), 480-1, http://www.ncbi.nlm.nih.gov/pubmed/13355476 (Aug 1956).

23. Dunsky, E. H., Zweiman, B., Fischler, E. \& Levy, D. A. Early effects of corticosteroids on basophils, leukocyte histamine, and tissue histamine. J Allergy Clin Immunol 63(6), 426-32, https://doi.org/10.1016/0091-6749(79)90217-3 (Jun 1979).

24. Wollin, A., Navert, H. \& Bounous, G. Effect of intestinal ischemia on diamine oxidase activity in rat intestinal tissue and blood. Gastroenterology 80(2), 349-55, https://www.ncbi.nlm.nih.gov/pubmed/6778762 (Feb 1981).

25. Lorenz, W. et al. Histamine release during orthotopic homologous liver transplantation in pigs. Eur. Surg. Res. 5(1), 11-20, http:// www.ncbi.nlm.nih.gov/pubmed/4119938 (1973).

26. D’Agostino, L. et al. Metabolic fate of plasma diamine oxidase: evidence of isolated and perfused rat liver uptake. Digestion 34(4), 243-50, http://www.ncbi.nlm.nih.gov/pubmed/3091435 (1986).

27. Velicky, P. et al. Pregnancy-associated diamine oxidase originates from extravillous trophoblasts and is decreased in early-onset preeclampsia. Sci Rep 8(1), 6342, https://doi.org/10.1038/s41598-018-24652-0 (Apr 20, 2018).

28. Klocker, J., Drasche, A., Sattler, J., Bodner, E. \& Schwelberger, H. G. Postheparin plasma diamine oxidase activity and the anticoagulant effect of heparin. Inflamm Res 49(Suppl 1), S53-4, http://www.ncbi.nlm.nih.gov/pubmed/10864420 (Apr 2000).

29. Wolvekamp, M. C. \& de Bruin, R. W. Diamine oxidase: an overview of historical, biochemical and functional aspects. Dig Dis 12(1), 2-14, http://www.ncbi.nlm.nih.gov/pubmed/8200121 (Jan-Feb 1994).

30. Teien, A. N. Heparin elimination in patients with liver cirrhosis. Thromb Haemost 38(3), 701-6, http://www.ncbi.nlm.nih.gov/ pubmed/579516 (Oct 31, 1977).

31. McKee, R. F., Hodson, S., Dawes, J.,Garden, O. J. \& Carter, D. C. Plasma concentrations of endogenous heparinoids in portal hypertension. Gut 33(11), 1549-52, http://www.ncbi.nlm.nih.gov/pubmed/1452082, http://gut.bmj.com/content/33/11/1549.full. pdf (Nov 1992).

32. Kettner, S. C. et al. Endogenous heparin-like substances significantly impair coagulation in patients undergoing orthotopic liver transplantation. Anesth Analg 86(4), 691-5, http://www.ncbi.nlm.nih.gov/pubmed/9539584 (Apr 1998).

33. Felli, A. et al. Different Heparin Contents in Prothrombin Complex Concentrates May Impair Blood Clotting in Outpatients With Ventricular Assist Devices Receiving Phenprocoumon. J Cardiothorac Vasc Anesth 30(1), 96-101, https://doi.org/10.1053/j. jvca.2015.08.012 (Jan 2016)

34. Cruz, R. J. et al. Hepatosplanchnic vasoregulation and oxygen consumption during selective aortic blood flow reduction and reperfusion. J Surg Res 171(2), 532-9, https://doi.org/10.1016/j.jss.2010.05.037 (Dec 2011).

35. Tsunooka, N. et al. Ischemia of the intestinal mucosa during cardiopulmonary bypass. J ArtifOrgans 6(2), 149-51, http://www.ncbi. nlm.nih.gov/pubmed/14621695 (2003).

36. Lorenz, W. et al. Significance and causes of histamine release during orthopic homologous liver transplantation in the pig. Agents Actions 3(1), 2-11, http://www.ncbi.nlm.nih.gov/pubmed/4123705 (May 1973).

\section{Acknowledgements}

The authors thank all of the participants and health care professionals for their contributions to this study. We further wish to acknowledge Prof. Andrea Quaroni, Cornell University, USA, for providing the hybridoma cell line. This work was supported by the Austrian Science Funds SFB54-P04. The Austrian Science Fund was neither involved in the conduct of the study nor in the writing of the report or in the decision to submit the article for publication. Financial support for this work was also provided by departmental funds.

\section{Author contributions}

J.S. and B.J. designed and conducted the study, collected and analyzed data, and drafted the first version of the manuscript. G.B. and J.B.-S. helped to conduct the study, collected data, and actively contributed to the content of the manuscript. T.B., L.K., P.W., J.M., P.J.-S. and M.S. contributed to the content of the manuscript, and edited the final version the manuscript. J.M., J.B.-S. and P.F. helped to design and conduct the study, collected and analyzed data, actively contributed to the content of the manuscript, and edited the final version the manuscript. All authors approved the final version of the manuscript.

\section{Competing interests}

The authors declare no competing interests.

\section{Additional information}

Correspondence and requests for materials should be addressed to B.J.

Reprints and permissions information is available at www.nature.com/reprints.

Publisher's note Springer Nature remains neutral with regard to jurisdictional claims in published maps and institutional affiliations.

(c) (i) Open Access This article is licensed under a Creative Commons Attribution 4.0 International (c) License, which permits use, sharing, adaptation, distribution and reproduction in any medium or format, as long as you give appropriate credit to the original author(s) and the source, provide a link to the Creative Commons license, and indicate if changes were made. The images or other third party material in this article are included in the article's Creative Commons license, unless indicated otherwise in a credit line to the material. If material is not included in the article's Creative Commons license and your intended use is not permitted by statutory regulation or exceeds the permitted use, you will need to obtain permission directly from the copyright holder. To view a copy of this license, visit http://creativecommons.org/licenses/by/4.0/.

(C) The Author(s) 2020 